\title{
DIVERSIDAD SEXUAL, DONACIÓN DE SANGRE Y NO DISCRIMINACIÓN EN CONTEXTO DE PANDEMIA* Avances en la jurisprudencia de Brasil
}

\author{
SEXUAL DIVERSITY, BLOOD DONATION AND NON-DISCRIMINATION IN \\ THE CONTEXT OF PANDEMIC \\ Advances in Brazilian jurisprudence
}

Martha Lisiane Aguiar Cavalcante**

\begin{abstract}
RESUMEN: Este trabajo propone analizar los avances en la jurisprudencia del Tribunal Constitucional de Brasil sobre el control de constitucionalidad de la norma que establece la prohibición de donación de sangre de hombres que hayan tenido relaciones sexuales con otros hombres. La necesidad de ampliación de donadores de sangre por causa de la pandemia de COVID-19 desencadenó un proceso de presión social por parte de instituciones democráticas y de organizaciones no gubernamentales, que culminó con la votación de la ADI 5543 y con la aplicación interna de los principios de igualdad y no discriminación y de normas internacionales de derechos humanos.
\end{abstract}

ABSTRACT: This paper proposes to analyze the advances in the jurisprudence of the Constitutional Court of Brazil on the constitutional control of the norm that establishes the prohibition of blood donation by men who have had sexual relations with other men. The need to expand blood donors due to the covid-19 pandemic triggered a process of social pressure by democratic institutions and non-governmental organizations, which culminated in the vote of IDA 5543 and the domestic application of the principles of equality and nondiscrimination and international human rights standards.

PALABRAS CLAVE: Diversidad sexual, discriminación, pandemia, avance jurisprudencial.

KEYWORDS: Sexual diversity, discrimination, pandemic, jurisprudential advances.

Fecha de recepción: 15/02/2021

Fecha de aceptación: 15/02/2021

doi: https://doi.org/10.20318/universitas.2021.6204

\footnotetext{
* Trabajo presentado en el II Congreso de la Asociación Estudiantes y Egresadas/os Instituto de Derechos Humanos Gregorio Peces-Barba.

${ }^{* *}$ Defensora pública en Brasil. Experta en Derecho Constitucional. Magíster en Estudios Avanzados en Derecho Humanos de Universidad Carlos III de Madrid. Email: martha.cavalcante@gmail.com.
} 


\section{1.- INTRODUCCIÓN}

Las personas LGBTI han sido víctimas de discriminación, estigmatización, violencia y violaciones de derechos humanos debido a las diversidades sexual y corpórea ${ }^{1}$. El trabajo está delimitado al análisis de la Portaria no 158/2016, de 4 de febrero de 2016, ${ }^{2}$ que redefine el reglamento técnico de procedimientos de donación de sangre y considera inaptos por doce meses a los hombres que hayan tenido relaciones sexuales con otros hombres $y / 0$ sus parejas sexuales. ${ }^{3}$

El dispositivo no menciona como criterio de selección de donantes de sangre el método que presenta mayor eficacia para evitar el contagio de SIDA que es el uso de preservativos en las relaciones sexuales.

Para hombres que tengan relaciones sexuales con otros hombres la medida normativa exige absoluta ausencia de cualquier relación sexual por un período mínimo de un año. Ya para los hombres heterosexuales es suficiente que hayan tenido sexo con pareja fija en los 12 (doce) meses anteriores a la donación, aunque sin uso de preservativos (que no es una condición normativa).

La discriminación de este grupo en situación de vulnerabilidad radica en el hecho de que cualquier relación sexual con otro hombre se convierte en obstáculo insuperable para la donación de sangre, aunque haya ocurrido con pareja fija y con el uso de preservativo, además de no considerar la evaluación concreta de la sangre a través de los exámenes.

Esta diferenciación que ignora las prácticas sexuales concretas y se basa en la orientación sexual de las personas involucradas clasifica a hombres gais y bisexuales como grupo de riesgo, lo que supone un concepto discriminatorio que no debe orientar la actuación estatal.

El objetivo del trabajo es investigar la Portaria no 158/2016 del Ministerio de Sanidad de Brasil, a través de los parámetros internacionales y nacionales de discriminación, así como el reciente cambio en la teoría de derechos y en la jurisprudencia debido a la necesidad de ampliar el número de donadores de sangre por causa de la pandemia.

\footnotetext{
${ }^{1}$ La investigación adoptó la sigla LGBTI porque es la más utilizada en el sistema interamericano de derechos humanos, sin olvidar de la multiplicidad de términos más amplios como LGTBIQA+ (lesbianas, gais, transexuales, bisexuales, intersexos, queer, asexual y otros).

2 Portaria no 158/2016, Ministerio de Sanidad de Brasil es el instrumento normativo investigado y tiene la función de reglamentar una legislación

<http://bvsms.saude.gov.br/bvs/saudelegis/gm/2016/prt0158_04_02_2016.html> Última consulta em 09 enero 2021.

${ }^{3}$ Ibid, artículo 64: Se considerará no apto temporalmente durante 12 (doce) meses el candidato que haya sido expuesto a cualquiera de las siguientes situaciones: IV los hombres que hayan tenido relaciones sexuales con otros hombres y/o sus parejas sexuales.
} 
El capítulo dos plantea la orientación sexual como condición social de vulnerabilidad, a través del análisis de conceptos importantes y de la realidad de protección normativa o criminalización de las personas LGBTI en países en el mundo.

La Asociación Internacional de Gais, Lesbianas, Bisexuales, Transexuales e Intersexuales (MUNDO ILGA) ha divulgado, en el año de 2019, el Informe Homofobia de Estado con quinientas páginas sobre diversos aspectos de la legislación protectora o discriminatoria sobre el tema. A pesar de algunos avances con relación al informe de 2018, ILGA MUNDO ha concluido que en uno de cada tres países es peligroso mostrarse como persona LGTBI.

El capítulo tres examina la discriminación en los instrumentos normativos internacionales y analiza la norma que restringe la donación de sangre de hombres que hayan tenido relaciones sexuales con otros hombres y/o sus parejas sexuales (Portaria n. ${ }^{\circ}$ 158/2016 del Ministerio de Sanidad de Brasil).

En el año de 2020, la norma analizada ha sido sometida al control de constitucionalidad en Brasil con parámetro en la dignidad humana, respeto, diversidad, autonomía y no discriminación. El capítulo cuatro plantea los argumentos de hecho y jurídicos del proceso (ADI 5543), la participación de la sociedad, la influencia de la política de extrema derecha y de la pandemia de COVID-19 en la interpretación sobre igualdad y no discriminación ${ }^{4}$.

\section{2.- ORIENTACIÓN SEXUAL COMO CONDICIÓN SOCIAL DE VULNERABILIDAD}

Es importante señalar que la heteronormatividad es un régimen político, económico, social y filosófico que genera diversas violencias contra las personas que no siguen un determinado patrón de sexualidad, de género, de prácticas y de deseos relativos a la heterosexualidad. ${ }^{5}$

Daniel J. García López plantea la heteronormatividad como "un conjunto de normas heterónomas (impuestas al sujeto desde fuera), generales y, en ocasiones, invisibles sobre la heterosexualidad". El autor explica el proceso de institucionalización del cuerpo ideal y qué normas son naturales y objetivas. De ahí, la verdad de un grupo se impone a otros grupos de manera invisible y desapercibida. ${ }^{6}$

Las personas que no están de acuerdo con la normatividad de dicho régimen han sufrido un proceso de discriminación y exclusión de derechos porque la diversidad sexual ha sido asociada a patologías.

\footnotetext{
${ }^{4}$ ADI significa acción directa de constitucionalidad que es una herramienta para controlar leyes u otras normativas que violan la Constitución.

5 R. Lucas Platero Méndez y María Rosón Villena y Esther Ortega Arjonilla, Barbarismos queer y otras esdrújulas, 228.

${ }^{6}$ Daniel J. García López, Sobre el derecho de los hermafroditas, 44.
} 
Michel Foucault señala que la represión al sexo tiene un fondo histórico y político como desarrollo del capitalismo, del orden burgués y de la excesiva explotación de la fuerza de trabajo, que no toleraba perder tiempo de producción para buscar placer en el sexo.7 Así, con el tránsito a la modernidad, la sociedad fue controlada en dos estrategias del biopoder: a) disciplina, que reflexiona sobre "la táctica, el aprendizaje, la educación, el orden de las sociedades", como el ejército y las escuelas; b) regulaciones de población, a través de "la demografía, la estimación de la relación entre recursos y habitantes, los cuadros de las riquezas y su circulación, de las vidas y su probable duración". ${ }^{8}$

El autor destaca que:

La psiquiatrización del placer perverso en que el instinto sexual fue aislado como instinto biológico y psíquico autónomo; se hizo el análisis clínico de todas las formas de anomalías que pueden afectarlo; se le prestó un papel de normalización y patologización de la conducta entera; por último, se buscó una tecnología correctiva de dichas anomalías. ${ }^{9}$

El conocimiento médico se ha encargado de corregir las supuestas anomalías (patologías) y el sistema jurídico ha cumplido el rol de sancionar y punir las conductas criminales. Así, el derecho ha estudiado y castigado "Ios comportamientos que se alejen de lo que fisiológicamente debe realizar un cuerpo", como lo que se aleja se la función reproductiva entre el hombre y la mujer, la base del sistema jurídico heteronormativo y binario de las sociedades. ${ }^{10}$

Para acercarse del tema LGBTI es importante señalar el sistema binario sexo/género y la teoría queer. Damian A Gonzalez-Salzberg plantea que las normas de género que dividen a las personas en hombres y mujeres pueden considerarse el sistema normativo más antiguo para clasificar a las personas. Sin embargo, como con todas las demás órdenes reglamentarias, se puede argumentar que estas normas no son intemporales, sino una creación histórica. ${ }^{11}$

El concepto binario sexo/género es "un pensamiento hegemónico que representa la realidad desde una concepción dicotómica y opositiva, basada en la idea genérica de creación del mundo y de complementariedad de los sexos, que ha consolidado una jerarquía sexual". ${ }^{12}$

Judith Butler plantea que, si el género está relacionado a los significados culturales que son atribuidos al cuerpo sexuado, entonces no se puede afirmar que un género únicamente sea

\footnotetext{
${ }^{7}$ Michel Foucault, Historia de la sexualidad I: la voluntad de saber, 7.

8 ibíd., 81.

9 Foucault, Historia de la sexualidad I: la voluntad de saber, 63.

10 Daniel J. García López, Sobre el derecho de los hermafroditas, 48.

${ }^{11}$ Damian A Gonzalez-Salzberg, Sexualitty and Transexualitty under the European Convetion on Human Rights, 14.

12 R. Lucas Platero Méndez y María Rosón Villena y Esther Ortega Arjonilla, Barbarismos queer y otras esdrújulas, 46.
} 
producto de un sexo. Llevada hasta su límite lógico, la distinción sexo/género muestra una discontinuidad radical entre cuerpos sexuados y géneros culturalmente construidos. ${ }^{13}$

La autora destacó que la supuesta distinción entre las categorías de hombres y mujeres como consecuencia de la existencia de dos sexos biológicos (hombre/mujer) era insostenible, ya que no existe una relación causal entre el sexo y el género. Así, ella propuso que el carácter cultural del género y el sexo, junto con la ausencia de una relación causal necesaria entre esos conceptos, muestra que las concepciones binarias de género y sexo son en realidad innecesarias. ${ }^{14}$

Como consecuencia de dicha construcción histórica y normativa, las personas han sido identificadas y encajadas en uno de los dos sexos (hombre/mujer), con una supuesta consecuencia directa en uno de los dos géneros (masculino/femenino) y una expectativa de orientación sexual direccionada al sexo contrario al suyo, sin considerar la diversidad sexual que también incluye a personas gais, lesbianas, transexuales, transgénero, intersex, bigénero, géneros fluidos, tercer sexo, etc. ${ }^{15}$

Por supuesto, toda esta diversidad ha sido patologizada por la medicina a través del estigma y construcción de estereotipos que van más allá de las ciencias médicas y afectan a la protección de derechos por el Estado, vulnerando la propria condición de ser humano.

Con relación a este tema, María del Carmen Barranco Avilés explica que:

la vulnerabilidad arranca de la construcción de estereotipos que condicionan las relaciones sociales y generan opresión, lo que a su vez implica que existen grupos de personas (los llamados vulnerables) que se encuentran en una posición de desventaja en relación con otros miembros de la sociedad, y que esta desventaja se justifica y se perpetúa por la incidencia de una ideología que la justifica en diferencias biológicas, naturales o inevitables con respecto a lo establecido como "norma" que se valoran negativamente. ${ }^{16}$

Boaventura de Sousa Santos entiende que la opresión y la exclusión de ciertos grupos de personas son consecuencias del colonialismo del poder, del capitalismo y del patriarcado. ${ }^{17}$

\footnotetext{
13 Judith Butler, El género en disputa, El feminismo y la subversión de la identidad, 54.

${ }^{14}$ Gonzalez-Salzberg, Sexualitty and Transexualitty under the European Convetion on Human Rights, 15.

15 Mirabilia Pandora, iImparables! Feminismos y LGBT+, 160.

16 María del Carmen Barranco Avilés, Condición humana y derechos humanos. Algunas claves filosóficas para un modelo contemporáneo de derechos, 27.

17 Boaventura de Sousa Santos, Construyendo las Epistemologías del Sur: para un pensamiento alternativo de alternativas, 326.
} 
Iris Marion Young plantea el siguiente concepto de opresión actualizado por los movimientos sociales emancipatorios feministas, socialistas, activistas indígenas, activistas gais y lesbianas:

la opresión designa las desventajas e injusticias que sufre alguna gente no porque un poder tiránico lo coaccione, sino por las prácticas cotidianas de una sociedad liberal (...) La opresión se refiere también a los impedimentos sistemáticos que sufren algunos grupos y que no necesariamente son el resultado de la voluntad de un tirano. Sus causas están insertas en normas, hábitos y símbolos que no se cuestionan, en los presupuestos que subyacen las reglas constitucionales y las consecuencias colectivas de seguir estas reglas. ${ }^{18}$

A partir de este cambio de perspectiva, la opresión pasa a ser percibida de forma estructural y sistémica, permeando las diversas relaciones sociales. El desarrollo del concepto de grupo social de esta autora también es una herramienta importante para consolidar la lucha de un colectivo que se aproxima por la diversidad sexual y por la discriminación y violencia generada como consecuencia. Iris Marion Young plantea que:

Un grupo social es un colectivo de personas que se diferencia de otros grupos por sus formas culturales, prácticas o modos de vida. Los miembros de un grupo tienen afinidades específicas debido a sus experiencias o formas de vida similares, lo cual los lleva a asociarse entre sí más que con aquellas otras personas que no se identifican en el grupo o que lo hacen de otro modo. ${ }^{19}$

Para contextualizar la vulnerabilidad y la opresión estructural generada a la comunidad LGTBI, se utilizaron datos recopilados en el último Informe de Homofobia de Estado de la Asociación Internacional de Lesbianas, Gais, Bisexuales, Trans e Intersex (ILGA WORLD), que es una federación mundial de más de 1.600 (mil seiscientos) organizaciones que pertenecen a más de 150 (ciento cincuenta) países.

El Informe de 2019 analiza que 123 (ciento veinte y tres) países tienen protección contra discriminación por orientación sexual. En algunos países la protección tiene fuerza y en otros es limitada. ${ }^{20}$ Es necesario destacar la trágica cifra que 70 (setenta) países que aún criminalizan actos sexuales consentidos entre adultos del mismo sexo.

El nivel de criminalización también es variado. Por ejemplo, 11 (once) países aún castigan estos actos sexuales con pena de muerte, 26 (veintiséis) países establecen la condena máxima en diez años de

\footnotetext{
18 Iris Marion Young, Justice and the Politics of Difference, 77.

19 ibíd., 77.

20 Asociación Internacional de Lesbianas, Gais, Bisexuales, Trans e Intersex (MUNDO ILGA): Lucas Ramón Mendos, Homofobia de Estado 2019, p. 16.
} 
prisión o cadena perpetua y en 31 (treinta y un) países se castiga con hasta ocho años de prisión. ${ }^{21}$

Desde su primera edición en 2006, este Informe ha sido un recurso fundamental en acceder a la información básica sobre la legislación que afecta a las personas en base a su orientación sexual y los cambios en las leyes a nivel mundial.

La agenda de avances contra la discriminación de personas LGBTI es muy variada. En algunos países se busca el derecho más fundamental a la vida. En otros países, la lucha está dirigida a la regulación del derecho al matrimonio igualitario y a la adopción conjunta por parejas del mismo sexo, a la prohibición de las terapias de conversión, contra la responsabilidad por delitos cometidos con base en la orientación sexual, etc. ${ }^{22}$

Este trabajo delimita la investigación sobre derechos LGBTI y no discriminación, al examen de determinada normativa de Brasil que establece la prohibición segregativa de donar sangre a los hombres que hayan tenido relaciones sexuales con otros hombres en Brasil.

El tercer capítulo analiza el tema a la luz de los principios generales del derecho internacional y de los instrumentos internacionales. Enseguida, el capítulo cuatro examina el impacto de la pandemia de COVID-19 en la jurisprudencia de Brasil.

\section{3.- PORTARIA N.0 158/2016 DEL MINISTERIO DE SANIDAD DE BRASIL E INSTRUMENTOS INTERNACIONALES DE IGUALDAD Y NO DISCRIMINACIÓN}

La norma analizada es la Portaria no. $158 / 2016$ que establece, en el art. 64, que se considerará no apto a donar sangre temporalmente durante 12 (doce) meses el candidato que haya sido expuesto a: IV - los hombres que hayan tenido relaciones sexuales con otros hombres y/o sus parejas sexuales.

La norma investigada no exige el uso de preservativo que es el método más indicado para evitar la contaminación por VIH. Además, solo exige de los hombres heterosexuales que tengan pareja fija, lo que demuestra un criterio no razonable e injustificado para discriminar hombres gais y bisexuales.

La orientación sexual es considerada por la norma como un factor de riesgo, aunque el procedimiento de donación de sangre exija exámenes específicos para evaluar las posibles enfermedades en la sangre, a través del NAT (prueba de ácido nucleico), con la reducción de la llamada ventana inmunológica de 60 para 10 días.

La norma analizada vulnera los instrumentos internacionales de protección contra la discriminación que se analizarán a continuación. Los instrumentos internacionales examinados son Los Principios de

21 ibíd. 
Yogyakarta, el Pacto Internacional de Derechos Económicos, Sociales y Culturales y la Convención Americana sobre Derechos Humanos.

Los sistemas normativos han cumplido durante siglos un rol social, político y jurídico para mantener la discriminación y estigmatización de la diversidad sexual. Sin embargo, a través del desarrollo de los derechos humanos, especialmente con la internacionalización y especificación, los sistemas universales y regionales de derechos humanos han protagonizado un importante cambio en la legislación y jurisprudencia de los Estados.

La humanización del derecho internacional se sitúa en la historia después de la Segunda Guerra Mundial, a través del desarrollo de un sistema de "reconocimiento internacional de derechos a las personas, por el respeto a su dignidad, a la libertad, a la seguridad e igualdad entre hombres y mujeres". ${ }^{23}$

El concepto de derechos humanos está relacionado con historicidad, tránsito de la modernidad y procesos de evolución histórica: positivación, generalización, internacionalización y especificación. Los dos últimos son especialmente relevantes para contextualizar la protección de personas LGBTI en el marco del sistema internacional de derechos humanos.

Gregorio Peces-Barba plantea que la positivación de los derechos naturales se justifica en el iusnaturalismo racionalista por significar una mayor eficacia e, indirectamente, la ideología contractualista caracterizada por la vinculación entre Derecho y poder. ${ }^{24}$

El marco evolutivo siguiente es la generalización que tiene como punto de partida considerar "ciertas circunstancias que pueden afectar a todos los seres humanos y que suponen que la dignidad se pueda poner en peligro". A saber, el "proceso de generalización implica la ampliación de la titularidad de los derechos, que se manifiesta, de modo muy claro, en la extensión de la ciudadanía". ${ }^{25}$

De ahí, la generalización "es consecuencia de la dimensión igualitaria con la que lingüísticamente se formulan en la última fase del modelo americano y sobre todo en la Declaración francesa, al afirmar el artículo primero que todos los hombres nacen y permanecen libres e iguales en derechos". ${ }^{26}$

Por otro lado, la especificación es el proceso histórico más reciente y se caracteriza por una ruptura con el clásico modelo abstracto y racional de búsqueda de un consenso social para limitar

23 Florabel Quispe Remón, La protección de los derechos humanos en el sistema interamericano: su evolución y una visión actual, 226.

24 Gregorio Peces-Barba Martínez, Curso de derechos fundamentales. Teoría General, 156.

25 Avilés, Condición humana y derechos humanos. Algunas claves filosóficas para un modelo contemporáneo de derechos, 13.

26 Declaración francesa, artículo $1^{\circ}$. Los hombres nacen y permanecen libres e iguales en derechos. Las distinciones sociales solo pueden fundarse en la utilidad común. 
el poder del Estado absolutista y garantizar las ideas prevalentes en la participación de los ciudadanos, a través de conceptos mayoritarios.

En efecto, los derechos han sido justificados por consenso en cuanto a limitaciones del poder estatal y a la participación de los ciudadanos a través de un consenso democrático pautado en las mayorías. ${ }^{27}$ Al contrario, el marco de la especificación es la protección de la dignidad de personas en situaciones de vulnerabilidad, lo que generalmente es una situación contra la mayoría.

Después de la breve aproximación a la evolución de los derechos humanos, se toma nota de algunos instrumentos internacionales importantes a la investigación sobre igualdad y no discriminación.

Ciertamente se destacan Los Principios de Yogyakarta, que establecen normas de derechos humanos y su aplicación a las cuestiones relativas a la orientación sexual y la identidad de género. Ellos afirman la obligación primordial que cabe a los Estados en cuanto a la implementación de los derechos humanos. ${ }^{28}$

Los Principios de Yogyakarta fueron elaborados por expertos y establecen conceptos y normas de derechos humanos para aplicación a las cuestiones relativas a la orientación sexual y la identidad de género. Según este documento, la orientación sexual se refiere a la "capacidad de cada persona de sentir una profunda atracción emocional, afectiva y sexual por personas de un género diferente al suyo, o de su mismo género, o de más de un género, como también es la capacidad de mantener relaciones íntimas y sexuales con estas personas". ${ }^{29}$

La identidad de género se define como "la vivencia interna e individual del género tal como cada persona la siente profundamente, que puede corresponder o no con el sexo asignado al momento del nacimiento. Está incluida también la vivencia personal del cuerpo y otras expresiones de género, incluyendo la vestimenta, el modo de hablar y los modales". ${ }^{30}$

Sobre los derechos a la igualdad y a la no discriminación, el instrumento plantea que todas las personas tienen derecho al disfrute de todos los derechos humanos, sin discriminación por motivos de orientación sexual o identidad de género. Todas las personas tienen derecho a ser iguales ante la ley y tienen derecho a igual protección por parte de la ley, sin ninguna de las discriminaciones mencionadas, ya sea que el disfrute de otro derecho humano también esté afectado o no. ${ }^{31}$

27 Martínez, Curso de derechos fundamentales. Teoría General, 180.

${ }^{28}$ LOS PRINCÍPIOS DE YOGYAKARTA,

<http://www.clam.org.br/uploads/conteudo/principios_de_yogyakarta.pdf > última consulta en 09 de enero de 2021.

29 ibíd.

30 ibíd.

31 ibíd. 
La Convención Americana sobre Derechos Humanos establece que los Estados Partes se comprometen a respetar los derechos y las libertades reconocidos en ella y a garantizar su libre y pleno ejercicio a toda persona que esté sujeta a su jurisdicción, sin discriminación alguna por motivos de raza, color, sexo, idioma, religión, opiniones políticas o de cualquier otra índole, origen nacional o social, posición económica, nacimiento o cualquier otra condición social. ${ }^{32}$

La orientación sexual no está expresamente prevista en la Convención y está encuadrada en cualquier otra condición social. No obstante, la Comisión Interamericana y la Corte Interamericana han reconocido la obligación de los Estados parte de no discriminar a personas LGBTI como consecuencia de la incorporación de la orientación sexual y de la identidad de género como "otra condición social" bajo el artículo 1.1 de la Convención Americana. ${ }^{33}$

La orientación sexual y la identidad o expresión de genero no están numeradas en los documentos básicos del sistema interamericano de derechos humanos. La protección de las diversidades sexual, de género y corporal ha evolucionado en el sistema regional, a través de la actuación de los órganos del Sistema Interamericano, que son la Comisión Interamericana de Derechos Humanos y la Corte Interamericana de Derechos Humanos. Las funciones de dichos órganos han sido esenciales para la ampliación del catálogo de derechos e inclusión de protección a derechos LGBTI en Convenciones más recientes.

Además, la Opinión Consultiva OC-24/17 plantea que no toda diferencia de trato será reputada discriminatoria, sino solo aquella que se base en criterios que no puedan ser racionalmente apreciados como objetivos y razonables, es decir, cuando no persigue un fin legítimo y no existe una relación razonable de proporcionalidad entre los medios utilizados y el fin perseguido. ${ }^{34}$

Con relación al sistema universal de derechos humanos es necesario señalar el artículo 2.2 del Pacto Internacional de Derechos Económicos, Sociales y Culturales, que tampoco establece expresamente la protección a la no discriminación por orientación sexual que está encuadrada en otra condición social. ${ }^{35}$

\footnotetext{
32 Convención Americana Sobre Derechos Humanos <http://www.corteidh.or.cr/> última consulta 09 enero 2021.

33 COMISIÓN INTERAMERICANA DE DERECHOS HUMANOS, Violencia contra Personas Lesbianas, Gais, Bisexuales, Trans e Intersex en Américas, 12 de noviembre de 2015, para 40.

${ }^{34}$ CORTE INTERAMERICANA DE DERECHOS HUMANOS, OPINIÓN CONSULTIVA 24/2017<http://www.corteidh.or.cr/cf/Jurisprudencia2/busqueda_opiniones_consul tivas.cfm?lang=es $>$ última consulta 09 enero 2021.

35 PACTO INTERNACIONAL DE DERECHOS ECONÓMICOS, SOCIALES Y

CULTURALES, disponible en

https://www.ohchr.org/SP/ProfessionalInterest/Pages/CESCR.aspx.
} 
La Observación General no 20 del Comité de Derechos Económicos, Sociales y Culturales toma nota de la discriminación sistémica como:

Un conjunto de actitudes legales, políticas, prácticas o culturales predominantes en el sector público o privado que crean desventajas relativas para ciertos grupos y privilegios para otros grupos y existe en situaciones donde la discriminación está fuertemente arraigada en el comportamiento y la organización de la sociedad. ${ }^{36}$

Así, la norma investigada no está de acuerdo con los instrumentos internacionales porque está basada en criterios que no pueden ser racionalmente apreciados como objetivos y razonables, ya que existen exámenes para averiguar la salud de la sangre proveniente de donación, que es la manera científica más adecuada.

La discriminación está basada en el estereotipo de promiscuidad de los hombres que han tenido relaciones sexuales con otros hombres. Además, no existe una relación razonable de proporcionalidad entre los medios utilizados y el fin perseguido.

\section{4.- LA PANDEMIA DE COVID-19 Y LOS AVANCES EN LA JURISPRUDENCIA DEL TRIBUNAL CONSTITUCIONAL DE BRASIL}

La Constitución de Brasil, en el art. 10, establece que la República Federativa de Brasil se constituye en Estado Democrático de Derecho y tiene como fundamento la dignidad de la persona humana. ${ }^{37}$

No existe protección constitucional expresa a la diversidad de orientación sexual y los derechos de las personas LGBTI se ejercen con fundamento jurídico en el derecho a la igualdad del artículo $5^{\circ}$ de la Constitución. 38

Además, el artículo 30 define como objetivo fundamental la promoción del bien de todos, sin prejuicios de origen, raza, sexo, color, edad o cualquier otra forma de discriminación. Una vez más, la orientación sexual no está expresa, pero tiene protección como otra forma de discriminación. ${ }^{39}$

\footnotetext{
36 <https://www.ohchr.org>

${ }^{37}$ Constitución de

Brasil

<http://www.planalto.gov.br/ccivil_03/constituicao/constituicao.htm> última consulta 09 enero 2021.

38 ibíd., artículo 50: Todos son iguales ante la ley, sin distinción de cualquier naturaleza, garantizándose a los brasileños y a los extranjeros residentes en el País la inviolabilidad del derecho a la vida, a la libertad, a la igualdad, a la seguridad y a la propiedad.

39 ibíd., art. 30. Constituyen objetivos fundamentales de la República Federativa de Brasil: IV - promover el bien de todos, sin prejuicios de origen, raza, sexo, color, edad o cualquier otra forma de discriminación.
} 
La Portaria no. 158/2016 fue sometida, en 2017, al control de constitucionalidad del Supremo Tribunal Federal (STF) de Brasil, a través de la ADI 5543, cuando el ministro ponente del procedimiento Edson Fachin entendió que por su inconstitucionalidad por entender que:

la norma viola la forma de ser y de existir de estas personas y el fundamento del respeto a la diversidad y a la dignidad humana, y afrenta la autonomía de quien desea donar sangre, que no está limitada por razones médicas o científicas. ${ }^{40}$

El criterio científico más adecuado es la realización de los exámenes en la sangre donada. Además, si la legislación va a adoptar prácticas de riesgo la razonabilidad exige que estas estén relacionadas al acto sexual con la utilización de preservativo y no a la orientación sexual de las personas.

El análisis de la inconstitucionalidad de dicha prohibición comenzó en 2017, pero permaneció sin decisión durante muchos años, en que el proceso entró y salió de la agenda varias veces sin juicio debido a la difícil coyuntura política enfrentada con el ascenso de la extrema derecha al poder, con sus valores conservadores y discriminatorios.

El treinta de abril de 2020, ante la baja cantidad de sangre para transfusiones provocada por la pandemia del COVID-19, la Defensoría Pública de la Unión (DPU) pidió agilidad en el juicio de la propuesta ${ }^{41}$.

En el día ocho de mayo de 2020, finalmente los once ministros del STF pronunciaron sus votos $y$, por siete votos a cuatro, el Tribunal declaró inconstitucional y discriminatoria la norma investigada que impide la donación de sangre basada en determinada orientación sexual.

La sociedad civil ha contribuido en la decisión, a través del amicus curiae, con la representación de asociaciones LGTBI, Organización Mundial de Salud y Defensorías Públicas, garantizando un debate más amplio y democrático.

Simmy Larrat, presidente de la Asociación Brasileña de Gais, Lesbianas, Travestis y Transexuales, destacó la existencia de una inmensa parte de la población que practica sexo anal. Pero no se les pregunta a todas las personas que tienen la intención de donar sangre sobre la práctica sexual anal, solamente a una parte de ellas. Es por ello que él destaca que actualmente no hay justificación para este prejuicio. ${ }^{42}$

La declaración de inconstitucionalidad de dicha norma discriminatoria significa un cambio jurisprudencial del Tribunal Constitucional de Brasil, que aplicó los principios de igualdad y no

40 Supremo Tribunal Federal, ADI 5543 <http://portal.stf.jus.br> última consulta 09 enero 2021.

${ }^{41}$ Es la institución responsable por la promoción de derechos humanos en Brasil.

42 ibíd. 
discriminación para garantizar la protección de derechos de las personas LGBTI, de acuerdo con los instrumentos normativos internacionales y con la interpretación de la Corte Interamericana de Derechos Humanos.

La jurisprudencia del Supremo Tribunal Federal es un precedente para diversos países que aún discriminan la donación de sangre de personas LGBTI y se estima que propicia el aumento de un millón y medio de litros de sangre al mes, lo que supone el cuarenta por ciento $(40 \%)$ de la capacidad actual, en medio a la pandemia por COVID-19.

En efecto, la necesidad de ampliación del número de donadores de sangre por causa de la pandemia desencadenó un proceso de presión social por parte de instituciones democráticas (como la Defensoría Pública) y de organizaciones no gubernamentales LGBTI, que culminó con la colocación en pauta de la ADI 5543 para votación y la decisión favorable a la aplicación interna de los marcos internacionales de derechos humanos.

\section{5.- CONCLUSIÓN}

La comunidad LGBTI está en condición de vulnerabilidad porque en uno de cada tres países del mundo es peligroso mostrarse como miembro de dicho grupo social, so pena de sufrir violaciones de diversa índole, que pueden afectar incluso el derecho a la vida.

La opresión basada en el estereotipo y el prejuicio de promiscuidad de hombres que tienen relaciones sexuales con otros hombres crea un obstáculo estatal y una desventaja para donar sangre. La discriminación es sistémica, injustificada y no es razonable ya que la sangre donada se somete a exámenes científicos que testan VIH.

La Portaria no. 158/2016 presenta un absurdo trato discriminatorio por parte del Poder Público en función de la orientación sexual, lo que ofende la dignidad de los implicados y les priva de la posibilidad de ejercer la solidaridad humana con la donación de sangre.

Las restricciones impuestas son prácticamente prohibitivas y tan solo se basan en la orientación sexual y el género del candidato a la donación y no en las llamadas prácticas de riesgo, que pueden afectar a toda la población.

El Tribunal Constitucional de Brasil ha decidido la inconstitucionalidad de la norma analizada, de acuerdo con los parámetros de los instrumentos normativos internacionales de igualdad y no discriminación.

A pesar de la coyuntura política desfavorable con la presión de los valores conservadores de la extrema derecha, la necesidad generada por la baja de las existencias de sangre como consecuencia de la pandemia impulsó el juicio de la demanda que ya estaba suspendido hace tres años. La decisión del Poder Judicial representa 
otro avance importante contra la violación de los derechos LGBTI en el mundo.

\section{6.- BIBLIOGRAFÍA}

\section{1.- Libros}

Avilés M. Diversidad de situaciones y universalidad de los derechos (Dykinson, 2010).

Butler J. El género en disputa, El feminismo y la subversión de la identidad (Paidós, 1999).

Foucault M. Historia de la sexualidad I: la voluntad de saber (Siglo veintiuno de España editores, 1998).

Gonzalez-Salzberg D. Sexualitty and Transexualitty under the European Convetion on Human Rights (Hart, 2019).

López D, Sobre el derecho de los hermafroditas (Melusina 2015).

Martínez G. Curso de derechos fundamentales. Teoría General (Universidad Carlos III de Madrid, 1999).

Méndez $R$ y Villena $M$ y Arjonilla $E$. Barbarismos queer $y$ otras esdrújulas (edicions Bellaterra, 2017).

Mirabilia P y Guixé M. iImparables! Feminismos y LGBT+ (Astronave, 2018).

Remón F. La protección de los derechos humanos en el sistema interamericano: su evolución y una visión actual (Anuario Español de Derecho Internacional, 2016).

Santos B. Construyendo las Epistemologías del Sur: para un pensamiento alternativo de alternativas, (CLACSO, 2018).

Young I, Justice and the Politics of Difference (Princeton University Press, 2011).

\section{2.- Normas internacionales e informes}

Asociación Internacional de Lesbianas, Gays, Bisexuales, Trans e Intersex (MUNDO ILGA): Lucas Ramón Mendos, Homofobia de Estado 2019 (Ginebra: ILGA, marzo de 2019).

COMISIÓN INTERAMERICANA DE DERECHOS HUMANOS, Violencia contra Personas Lesbianas, Gay, Bisexuales, Trans e Intersex en Américas, 12 de noviembre de 2015.

COMITÉ DE DERECHOS HUMANOS, OBSERVACIÓN GENERAL 20, disponible en <https://www.ohchr.org>, última consulta en 09 de enero de 2021.

CONVENCIÓN AMERICANA SOBRE DERECHOS HUMANOS, disponible en <http://www.corteidh.or.cr/>, última consulta en 09 de enero de 2021.

CORTE INTERAMERICANA DE DERECHOS HUMANOS, OPINIÓN CONSULTIVA 24/2017, disponible en <http://www.corteidh.or.cr/cf/Jurisprudencia2/busqueda_opinio nes_consultivas.cfm?lang=es $>$, última consulta en 09 de enero 
de 2021.

LOS PRINCIPIOS DE YOGYAKARTA, disponible en $<$ http://www.clam.org.br/uploads/conteudo/principios_de_yogya karta>, última consulta en 09 de enero de 2021.

PACTO INTERNACIONAL DE DERECHOS ECONÓMICOS, SOCIALES Y CULTURALES, disponible en <https://www.ohchr.org/SP/ProfessionalInterest/Pages/CESCR.a spx>.

\section{3.- Legislación nacional}

CONSTITUCIÓN DE BRASIL, disponible en <http://www.planalto.gov.br/ccivil_03/constituicao/constituicao. htm>, última consulta en 09 de enero de 2021.

PORTARIA No 158, DE 4 DE FEVEREIRO DE 2016, MINISTÉRIO DE SANIDAD DE BRASIL, disponible en https://www.in.gov.br/web/dou/-/portaria-n-158-de-4-defevereiro-de-2016-22301274, última consulta en 09 de enero de 2021.

\section{4.- Jurisprudencia}

SUPREMO TRIBUNAL FEDERAL, ADI 5543, disponible en <http://portal.stf.jus.br>, última consulta en 09 de enero de 2021. 